\title{
Evaluating rainbow colour scheme in social data mapping
}

Izabela Golebiowska

University of Warsaw, Faculty of Geography and Regional Studies, Department of Geoinformatics, Cartography and Remote Sensing, i.golebiowska@uw.edu.pl

Keywords: rainbow colour scheme, thematic maps, choropleth map, social data, user study

\begin{abstract}
:
Colour is one of the most useful variables to be applied in map design for information visualization. The choice of colour scheme affects the visual appearance of the map, its aesthetics, and how pleasant it appears. Consequently, it may, potentially, impact data perception, which has been proved by various authors (e.g. Brewer et al. 1997, Brychtova and Çöltekin 2015). There are established guidelines regarding colour use in cartography, for example, in textbooks. It is a well-established rule that when presenting quantitative data, the various values of the mapped data should be mainly represented by varying the colour value. But a growing frequency in colour use that contradicts the rules of cartographic visualization has been observed. The case is particularly true of the rainbow colour schemes (Figure 1.), which are commonly applied in geovisualisation practices in thematic maps; in the past, mainly on isoline maps; and currently, also on choropleth maps. The schemes involve the use of varying hues for presenting quantitative data, typically via a gradation based on the electromagnetic spectrum, instead of the practice recommended in cartography of varying colour value. The scale is interpolated through the saturated, spectrally ordered hues: blue, cyan, green, yellow, and red. The result is a vivid, fully saturated colour map that looks like a rainbow.
\end{abstract}

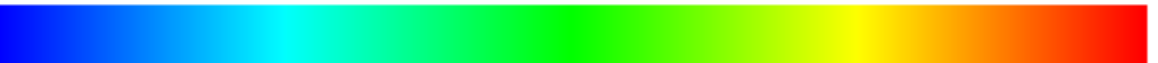

Figure 1. Rainbow colour scheme - one of the most commonly applied schemes in visualizations

This colour scheme is commonly considered to be a very poor way of representing data due to several problems. The disadvantages most often raised are as follows: (1) it is not perceptually ordered and there is no logical ordering (Monmonier 1991), which means there is no innate sense of higher or lower values; (2) it introduces sharp transitions between hues as the perceptual changes in the rainbow colours are not uniform (Moreland 2016), and which may be perceived as being a considerable transition in the mapped data; and (3) the middle values may be interpreted as extreme values since yellow has a highlighting effect, being perceived as brighter than the other colours.

The rainbow colour scheme is applied mainly in visualizing the quantitative data of various themes, both natural (weather maps, elevation) and socio-economic. The colour scheme can be found on on isoline maps; and currently, also on choropleth maps. Using rainbow colours for mapping natural phenomena such as temperature or elevation seems to be understandable, as they are presented using isolines. The use of spectral progression for relief maps is a convention of long standing; it is now well known through its regular appearance on school maps and atlas maps for the general public. However, applying this colour code to social data mapping using choropleth maps may not always be intuitive. Choropleth maps do not show the order of the colour on the face of the map, in contrast to isoline maps where colours are always in the same order on the map.

The issue of the incorrect use of the rainbow colour scheme in cartographic visualization was raised two decades ago. What is important is that Brewer (1997) raised doubts concerning the warnings that spectral hues were not ordered; and considered that this idea may no longer be valid due to users' continuous exposure to spectral graphics. There is a 'likelihood that the public is learning this code, through its use on most scientific visualizations that enter the public sphere.' (Brewer 1997: 217). Nowadays, this issue is still important with even more frequent use of spectral colour schemes in practice, often for non-diverging, sequential data. This makes the need for validating current practices more vital in order to verify if the commonly applied rainbow colour schemes can actually represent quantitative, non-diverging data on maps as effectively as the traditionally recommended sequential colour schemes. Further research is thus needed to validate the current common practices of using rainbow colours, and to establish evidence-based guidelines for colorcoding on thematic maps.

The aim of the study is to empirically evaluate the usability of the rainbow colour scheme for thematic maps. For this investigation choropleth maps presenting social data will be selected. Over 200 participants (high school students) will take part in the study and will be asked to solve different tasks using the presented maps. The participants will be dived into two groups, with each group solving the same tasks but using choropleth maps with differently designed colour 
scales: (1) a rainbow colour scheme, and (2) a colour scheme considered to be correct for non-diverging data, i.e., a sequential, value-varied colour scale. The study applies usability performance metrics, expressed as effectiveness (response correctness), efficiency (answer time), and preferences (users opinions). The participants will be given various tasks, ranging from reading quantities, to noting distributions and extracting patterns. Participants will also be asked to recall the studied maps. The tasks partially refer to the questions asked by Mersey (1990) in an in-depth evaluation of various colour schemes (including spectral). We thus want to compare this study's results with those collected over 30 years ago, and verify the assumption posed by Brewer (1997), that is, if nowadays, frequent exposure to rainbow maps results in improving usability of the rainbow colour scheme for maps, in a similar way - or to some extent - to the use of spectral colours for elevation maps.

By conducting this empirical investigation we hope to contribute to an increased understanding of how thematic geovisualisations should be designed in order to support users effectively. We also want to empirically verify the rules that have been established in cartographical theory, but which are often violated in practise. As Brewer (1997) noted, cartographers cannot stop people from selecting spectral schemes, but we can try to assess the consequences of applying such colour schemes to thematic maps.

This work was supported by the Polish National Science Centre [Grant number UMO-2016/23/B/HS6/03846], "Evaluation of cartographic presentation methods in the context of map perception and effectiveness of visual transmission" and by the Faculty of Geography and Regional Studies at the University of Warsaw. 